\title{
Collision tumors of the thyroid. A special clinical and pathological entity
}

\author{
Andreea Bojoga*, Laura Stănescu, Corin Badiu
}

"Carol Davila" University of Medicine and Pharmacy, Bucharest, Romania; "C.I. Parhon" National Institute of Endocrinology, Bucharest, Romania

\begin{abstract}
Thyroid collision tumors are rare entities that designate two histologically and morphologically distinct tumors that occur simultaneously or as metastases from other organs within the thyroid. Medullary and papillary carcinoma co-occurrence is the most frequent. Several theories tried to explain the pathogenic mechanisms underlining collision tumors, including the theory which assumes that one tumor predisposes the other, stem cell theory, and random effect theory, but their combination better explains the origin of these tumors. Hypotheses about common genetic behavior responsible for the pathogenesis have also been suggested, such as the involvement of germline mutation of RET (Rearranged during Transfection) protooncogene in medullary thyroid carcinoma and papillary thyroid carcinoma coexistence, but there is controversy on this topic. Management of thyroid collision tumors is challenging owing to the presence of two distinct tumors with different biological aggressiveness, treatments options, and prognosis, and needs to be individualized.
\end{abstract}

Keywords: cancer; thyroid; collision tumors

\section{Introduction}

Collision tumors are rare entities, occurring from two distinct histologic neoplasms, found in the same organ, but they are separated by normal tissue and have no histological admixture [1]. To date, synchronous carcinomas of the thyroid are described as a single tumor with mixed features, a single tumor with two distinct components, as two tumors separated by normal thyroid tissue or even collision metastases of these two tumors in the same cervical lymph node. These combinations can be classified into collision tumors, mixed tumors, and composite tumors.

Received: October 2021; Accepted after review: December 2021; Published: December 2021.

${ }^{*}$ Corresponding author: Andreea Bojoga, "Carol

Davila" University of Medicine and Pharmacy,

Bucharest, Romania

Email: andreeabojoga@yahoo.com
These terms are used to describe multiple synchronous tumors that express follicularderived and parafollicular cellular elements in the thyroid. However, these terms are not synonymous and are meant to indicate different entities.

These tumors arise simultaneously or as metastases from other organs, and are distinguished from mixed tumors which have a common cellular origin [2]. An example of mixed tumor of the thyroid is mixed medullary and follicular tumor of thyroid whereby there is morphological and immunohistochemical evidence of the both follicular and parafollicular tumor cells in the same lesion (MMFTC) (ICD-O code, 8346/3) [3].

Collision tumors are also distinct from composite tumors which usually occur as a result of a common driver mutation that induces different histological cellular populations in the same tumor [4].

Collision tumors have been described throughout the body, particularly within the thyroid gland. These account for about $1 \%$ of 
all thyroid tumors, with most literature describing co-occurring medullary thyroid carcinoma (MTC) and papillary thyroid carcinoma (PTC), or PTC coexisting with metastatic tumors $[1,2,5]$.

Synchronous thyroid carcinomas are extremely rare, accounting for $<1 \%$ of all thyroid tumors. This entity was initially described by Lamberg et al. in 1981 as a case of MTC with concurrent PTC.

Metastases to the thyroid gland are rare. The incidence is variable, up to $24 \%$ in autopsy studies, dependent on the extent of thyroid parenchymal sectioning, while clinical studies report an incidence of $2 \%-3 \%$ of all thyroid malignancies [6]. The most common primary sources reported are renal (clear cell renal cell carcinoma), breast, pulmonary, as well as squamous cell carcinomas (head and neck, esophageal, and lung) [2]. Less frequent primary sites are liver, adrenal gland, ovary, and gastrointestinal carcinomas (stomach, colon) [7].

Metastatic tumors of the thyroid may occur either by hematogenous spread from distant carcinomas or by direct extension of neoplasms from adjacent organs (squamous cell carcinoma, parathyroid carcinoma, soft tissue malignancies) [8].

The relatively low incidence is surprisingly given the rich vascularity of the thyroid which is second only to the adrenals in arterial blood flow volume per gram [1].

Two major hypotheses have been proposed: 1) rapid blood flow does not promote the adherence and seeding of metastases; 2) high oxygen saturation and iodine content are not favorable to the growth of malignant cells [9].

\section{Variants of thyroid collision tumors}

The most common thyroid collision tumor is that of differentiated thyroid cancer and medullary cancer coexistence [2, 10]. These are also the most common individual thyroid tumors, with PTC accounting for $90 \%$ of all thyroid cancer diagnosed, while follicular and medullary carcinomas account for only $4.4 \%$ and $1.5 \%$, respectively [11].
It is not uncommon to find papillary carcinomas in the background thyroid of patients who have surgery for medullary thyroid cancer, and the rate of incidental PTC in autopsy series ranges up to $35 \%$ and varies with the extent of parenchymal sectioning [12]. The largest case series of collision tumors of MTC and PTC was reported by Biscolla et al. who found that $13.8 \%$ of 196 patients with MTC had PTC in the form of collision tumors; micro PTC comprised $77 \%$ of these tumors [13]. The prevalence of these collision tumors is higher than the coexistence of PTC and multinodular goiter or Graves disease [2]. A similar incidence was reported by a population-based study, and this was found to be increasing [14].

PTC and MTC collision tumors seem to be more frequent in females ( $M / F$ ratio $1 / 2$ ), and to occur in the fifth, sixth, and seventh decade [15].

Collision tumors of PTC and follicular thyroid carcinoma (FTC) are thought to be rare and therefore there is a paucity of clinical data on this topic [5, 16]. However, Won Gu Kim et al. found that the occurrence rate of PTC in patients with follicular thyroid carcinoma was $19 \%$ (50 of the 268 FTC patients) and that most of PTC components were microcarcinomas [17].

FTC is the second most common type of thyroid carcinoma and tends to disseminate hematogenous leading to lung, bone and central nervous system metastases [5, 16]. Hematogenous and distant metastatic invasion is less common in PTC, and intrathyroidal and cervical multicentric disease is more frequent, pleading for lymphatic spread $[5,18]$.

Another reported case is an oncocytic thyroid carcinoma, coexisting with classical and hobnail variants of PTC [19]. There are only a few reported cases of collision tumors of the thyroid involving a metastasis from a distant tumor: coexistence of metastatic liposarcoma of the thigh to the thyroid with PTC [1], metastatic lung adenocarcinoma and PTC [18], metastatic thyroid osteosarcoma cooccurring with multifocal PTC [8]. Warman et al. reported a case of simultaneous occurrence of primary squamous cell carcinoma (SCC) of the thyroid gland and PTC [20]. Primary SCC of the thyroid gland is a rare 
and aggressive tumor and accounts for less than $1 \%$ of all thyroid neoplasms [21]. Sokoya et al. described an uncommon case of tracheal sarcomatoid carcinoma colliding with a papillary thyroid cancer [22].

\section{Diagnosis and treatment}

The variation in the reported incidence of collision tumors is partly attributed to different grossing protocols practiced, especially in nononcological centers, thus leading to underreporting of this entity, particularly the MTC component and especially pseudopapillary, follicular, and oncocytic subtypes of MTC [2]. An accurate diagnosis of the MTC component is crucial, having important consequences in the management and prognosis of these patients. The main presentation of MTC and PTC collision tumors is that of an anterior cervical mass, and patients may already present metastases [10]. SCC of the thyroid usually presents as a rapidly enlarging neck mass, often associated with dyspnea, dysphagia, and voice changes. Local invasion to adjacent structures and early metastatic dissemination are common [21]. Although rare, metastases to the thyroid gland should be considered when making the differential diagnosis of multiple thyroid nodules, especially in patients with a positive history of malignant tumors.

Cytology assessment of fine-needle aspiration of tumor rarely detects two distinct tumors, however more often it can identify a malignant pathology. It can be suggestive of metastasis but frequently simulates a primary thyroid tumor [7, 10]. Immunohistochemistry staining allows for the delineation of two coexisting separated tumors and should be included in further pathologic assessments of these collision tumors [18].

Imaging studies such as computed tomography scan, magnetic resonance imaging, positron emission tomographycomputed tomography with FDG (PET-CT) can be useful in the diagnosis of a secondary tumor. However, microcarcinomas of the PTC component may not be detected by imaging in the majority of cases. Thus, thoroughly pathological examination of the surgical specimen remains the only reliable diagnostic tool in most cases of thyroid collision tumors, and extensive microscopic assessment of individual tumors, including high-risk features, will help guide patient management of $[2,10]$.

Due to the paucity of reported literature on collision thyroid tumors, treatment guidelines are poorly defined. Management of thyroid collision tumors is challenging, as collision tumors usually contain components with different aggressiveness, treatments options, and prognosis. These tumors ought to be managed in a multidisciplinary framework and need to be individualized, considering biological aggressiveness and the stage of the tumor. Some authors recommend that each component of these collision tumors should be treated as a separate synchronous primary [2]. Others suggest that the most aggressive of the neoplasms should dictate the treatment, usually including surgical resection and adjuvant therapy [10]. The current guideline recommends that the adjuvant radioactive iodine treatment should be considered if the PTC component has any high-risk features. Biscolla et al. showed that the outcome of the MTC component of collision tumors was not influenced by the presence of the PTC and the consequent radioiodine treatment, but microcarcinomas comprised $77 \%$ of the PTC component of collision tumors [13].

In the simultaneous occurrence of MTC and PTC, calcitonin and thyroglobulin should be serially monitored for detecting tumor relapse; suppressive thyroid hormone therapy should be guided by the risk category of PTC tumor.

Treatment of thyroid metastases is usually palliative, but it should be individualized. Surgical resection when feasible can achieve local control or long-term cure [8]. Primary SCC of thyroid often associates airway compromise and palliative surgery is often recommended [23]. In addition, it is considered radiotherapy and chemotherapy-resistant, yet adjuvant postoperative radiotherapy has been associated with better survival rates [20].

Some suggest that the presence of two primary cancers behave more aggressively than individual tumors even though both tumors are differentiated, and present an increased risk of recurrence $[16,17]$. However, 
others have found that metastatic and survival rates are similar with matched singleton tumors [10].

\section{Pathogenic hypotheses}

The pathogenic mechanisms that cause collision tumors of the thyroid are still unclear, and case reports with extensive molecular characterization of these tumors disclose contradicting results regarding origin [2]. Several hypotheses have been suggested to explain the occurrence of collision tumors. The most widely accepted hypotheses are: i) one tumor predisposing the other tumor theory, ii) stem cell theory iii) random collision effect theory [19].

i) The first theory suggests that the occurrence of a tumor can instigate alterations in the microenvironment, such as blood flow, oxygen tension, and/or stem cell differentiation, which facilitate the development of a second malignancy [18]. Its growth may be further propagated by exposure to a common carcinogenic stimulus that triggers neoplastic transformation of the tumors involved, such as ionizing radiation [10, 24]. Pathophysiology of primary SCC of the thyroid gland has been debated as thyroid tissue does not normally contain squamous epithelium, excepting possible embryonic remnants involving branchial arch/thyroglossal duct [21]. It has been proposed that squamous metaplastic transformation is promoted by chronic inflammation which might transform into SCC [25]. Similarly, Darwish et al. have suggested that lymphocytic infiltration predispose to development of collision tumors of the thyroid [26]. However, this pathogenesis hypothesis fails to explain why these tumors are so rare, or to establish what conditions need to exist for one tumor to promote the development of a neighboring tumor and remains to be fully elucidated [10].

ii) The stem cell theory was first proposed by Ljunberg et al. in 1983 and suggested that tumors derive from a common stem cell [27]. This theory claims that such tumors occur due to the capability of cancer stem cells to differentiate into different cancer cell lines in the same organ or due to two distinct driver mutations in a common stem cell that lead to two separate tumors [2, 16]. In the case of MTC and PTC collision tumors, the assertion that these two components originate from a common stem cell, is derived from the ultimobranchial body which may serve as an embryological nest for such a stem cell [10]. This hypothesis was supported by coexpression of thyroglobulin and calcitonin in tumor cells which displayed a similar pattern of $X$ chromosomal inactivation and molecular alterations as loss of heterozygosity and mutational situation [28]. However, positive immunoreactivity for thyroglobulin of medullary carcinoma can be a result of thyroglobulin spillage, particularly at the edges of lesions, considering that invasive tumor cells overtake normal follicular structures [12]. If this theory is satisfying to explain the origin of collision carcinomas arising from follicular and parafollicular cells, this is not the case for other tumors, such as primary SCC of the thyroid. This "pluripotent precursor cell" theory fails to delineate collision tumors involving cell types of distinct embryological origin, which have been reported within the thyroid [10]. In addition, molecular evidence rather suggests that both components of these collision tumors were not derived from a common stem cell [29].

iii) Finally, random collision effect theory assumes that two separate and distinct tumor types such as MTC and a FTC or a MTC and a PTC, or PTC and a secondary thyroid metastasis, occur by chance. The components of collision tumors are thought to have independent origin either by de novo local genesis or coincidental seeding of a metastasis neighboring an unrelated primary tumor, the latter being more presumably in a patient with a history of malignancy $[2,10]$.

\section{Genetic considerations}

In the last decades, molecular studies have identified critical genetic alterations associated with the development of major thyroid cancer histological subtypes. Hypotheses about common genetic behavior responsible for the pathogenesis of different thyroid malignancies have been suggested. 
However, to date, no common gene mutation has been demonstrated.

In FTC and PTC collision tumors point mutations of the RAS oncogene such as (NRAS, H-RAS, and K-RAS), might be involved in the pathogenesis of these tumors, as these mutations have been reported in both PTC and FTC [16].

An attractive hypothesis proposed by some studies is that germline point mutation of the RET (Rearranged during Transfection) proto-oncogene had a potential pathogenetic role in the oncogenesis of MTC and PTC by tyrosine kinase activation [24, 28, 30].

Recent studies indicate the implication of germline point mutations in exon 13 and 14 in cases of concurrent PTC and MTC. Brauckhoff et al. described 104 patients with RET protooncogene germline mutations who underwent prophylactic surgery at their center. They found a $9.1 \%$ frequency of PTC in all patients with RET germline mutation in codon 790, 791 , and 804 [30]. Papi et al. reported a case of MTC and PTC in a patient with RET protooncogene germline mutation in codon 804 . No somatic mutations of the RET gene have been found in the tumor tissue in any case.

Oncogenically activating rearrangements of the RET proto-oncogene (RET/PTC) have been demonstrated in PTC, though have never been described in MTC [13, 31]. Mutations in different exons in RET protooncogene have been demonstrated in familial MTC as well as in multiple endocrine neoplasia (MEN) type 2 syndrome, and involvement of RET in the pathogenesis of these diseases is well-known [32]. With regards to collision tumors, Vantyghem et al. reported eleven cases of familial MTC-PTC, in which genetic studies failed to identify any RET mutations [33].

In cases of sporadic MTC, genetic polymorphism in the germinative lineage was studied and results were inconsistent [24]. Weinhaeusel et al. observed the presence of RET polymorphism in nearly all of 23 patients with sporadic MTC/C cell hyperplasia and synchronous PTC or follicular carcinomas [34].

Biscolla et al. reported similar rates of RET germline mutations in patients with simultaneous occurrence of MTC and PTC and patients with MTC alone, thus suggesting that these 2 tumors appeared to be separate entities; however, a shared pathogenic mechanism could not be excluded [13].

The possible common involvement of RET proto-oncogene in collision tumors was not confirmed by Rossi et al. who indicated a different genetic origin of the two coexisting neoplasms. This was the first report which documented two mutations, in the RET and BRAF genes, in MTC and PTC components, respectively, which are independent drivers of these tumors and have almost an insignificant chance of co-occurrence [2, 15]. Thus, the authors suggested that the occurrence of MTC and PTC was merely a fortuitous finding, and proposed designation of these neoplasms as "collision tumors" [10].

Ultimately, a combination of theories should be considered, such as in the case of a coincidental metastasis in which seeding's in the thyroid gland is favored by microenvironmental alterations [10, 16, 18]. None of the aforementioned theories can completely explain the pathogenesis of these tumors in all cases, and there is not enough evidence and understanding to support or rule out any of the hypotheses.

\section{Conclusions}

Collision tumors of the thyroid are rare entities, and co-occurrence of MTC and PTC constitutes the most common variant. PTC component in the form of microcarcinoma is not unusual and might be overlooked either on imaging, cytology, or histologic sampling. An adequate histologic assessment of these tumors is of utmost importance for diagnostic, staging, and therapeutic decisions. Pathogenetic mechanisms and molecular characterization of these tumors are still unclear and management of these tumors is challenging and poorly documented. Therefore, greater reporting with more conclusive evidence is needed to better understand and treat these tumors.

\section{Conflicts of interest}

There are no personal, financial, or other conflicts of interest to disclose. 


\section{References}

1. Brandwein-Gensler $M$, Urken $M$, Wang $B$. Collision tumor of the thyroid: a case report of metastatic liposarcoma plus papillary thyroid carcinoma. Head Neck. 2004; 26(7):637-641. https://doi.org/10.1002/hed.20024

2. Thomas A, Mittal N, Rane SU, et al. Papillary and medullary thyroid carcinomas presenting as collision tumors: a case series of 21 cases at a tertiary care cancer center. Head Neck Pathol. 2021; 15(4):1137-1146. https://doi.org/10.1007/s12105-021-01323-7

3. Volante M, Papotti M, Roth J, et al. Mixed medullary-follicular thyroid carcinoma. $A m \mathrm{~J}$ Pathol. 1999; 155(5):1499-1509. https://doi.org/10.1016/S0002-9440(10)65465$\mathrm{X}$

4. Walvekar RR, Kane SV, D'Cruz AK. Collision tumor of the thyroid: follicular variant of papillary carcinoma and squamous carcinoma. World J Surg Oncol. 2006; 4:65. https://doi.org/10.1186/1477-7819-4-65

5. Plauche V, Dewenter $\mathrm{T}$, Walvekar RR. Follicular and papillary carcinoma: a thyroid collision tumor. Indian J Otolaryngol Head Neck Surg. 2013; 65(Suppl 1):182-184. https://doi.org/10.1007/s12070-011-0450-0

6. Cichoń S, Anielski R, Konturek A, Barczyński $\mathrm{M}$, Cichon W. Metastases to the thyroid gland: seventeen cases operated on in a single clinical center. Langenbecks Arch Surg. 2006; 391(6):581-587. https://doi.org/10.1007/s00423-006-0081-1

7. Pastorello RG, Saieg MA. Metastases to the thyroid: potential cytologic mimics of primary thyroid neoplasms. Arch Pathol Lab Med. 2018; 143(3):394-399. https://doi.org/10.5858/arpa.2017-0570-RS

8. Koufopoulos N, Zacharatou A, Gouloumis A-R, et al. Metastatic thyroid osteosarcoma with concomitant multifocal papillary carcinoma presenting as a collision tumor. Cureus. 2021; 13(6):e15425.

https://doi.org/10.7759/cureus.15425

9. Chung AY, Tran TB, Brumund KT, Weisman RA, Bouvet M. Metastases to the thyroid: a review of the literature from the last decade. Thyroid Off J Am Thyroid Assoc. 2012; 22(3):258-268.

https://doi.org/10.1089/thy.2010.0154

10. Ryan N, Walkden G, Lazic D, Tierney P. Collision tumors of the thyroid: a case report and review of the literature. Head Neck. 2015; 37(10):E125-129.

https://doi.org/10.1002/hed.23936
11. Rossi ED, Pantanowitz L, Hornick JL. A worldwide journey of thyroid cancer incidence centred on tumour histology. Lancet Diabetes Endocrinol. 2021; 9(4):193-194. https://doi.org/10.1016/S2213-8587(21)000498

12. Sadow PM, Hunt JL. Mixed medullary-follicularderived carcinomas of the thyroid gland. $A d v$ Anat Pathol. 2010; 17(4):282-285. https://doi.org/10.1097/PAP.0b013e3181e4ab3 e

13. Biscolla RP, Ugolini C, Sculli M, et al. Medullary and papillary tumors are frequently associated in the same thyroid gland without evidence of reciprocal influence in their biologic behavior. Thyroid. 2004; 14(11):946-952. https://doi.org/10.1089/thy.2004.14.946

14. Wong RL, Kazaure HS, Roman SA, Sosa JA. Simultaneous medullary and differentiated thyroid cancer: a population-level analysis of an increasingly common entity. Ann Surg Oncol. 2012; 19(8):2635-2642. https://doi.org/10.1245/s10434-012-2357-8

15. Rossi S, Fugazzola L, De Pasquale L, et al. Medullary and papillary carcinoma of the thyroid gland occurring as a collision tumour: report of three cases with molecular analysis and review of the literature. Endocr Relat Cancer. 2005; 12(2):281-289. https://doi.org/10.1677/erc.1.00901

16. Pishdad R, Cespedes L, Boutin R, Jaloudi M, Raghuwanshi M. Coexistence of two different thyroid malignancies: a collision phenomenon. Cureus. 2020; 12(4):e7539. https://doi.org/10.7759/cureus.7539

17. Kim WG, Gong G, Kim EY, et al. Concurrent occurrence of medullary thyroid carcinoma and papillary thyroid carcinoma in the same thyroid should be considered as coincidental. Clin Endocrinol (Oxf). 2010; 72(2):256-263. https://doi.org/10.1111/j.13652265.2009.03622.x

18. Nabili V, Natarajan S, Hirschovitz S, Bhuta S, Abemayor E. Collision tumor of thyroid: metastatic lung adenocarcinoma plus papillary thyroid carcinoma. Am J Otolaryngol. 2007; 28(3):218-220. https://doi.org/10.1016/j.amjoto.2006.08.002

19. Toyoshima MTK, Domingues RB, Soares IC, et al. Thyroid collision tumor containing oncocytic carcinoma, classical and hobnail variants of papillary carcinoma and areas of poorly differentiated carcinoma. Arch Endocrinol Metab. 2021; 65(4):495-499. https://doi.org/10.20945/2359-3997000000389 
20. Warman M, Lipschitz N, Ikher S, Halperin D. Collision tumor of the thyroid gland: primary squamous cell and papillary thyroid carcinoma. ISRN Otolaryngol. 2011; 2011:582374. https://doi.org/10.5402/2011/582374

21. Soror NN, Shah $P$, Hemrock L, Bennett R. Primary squamous cell carcinoma of the thyroid: a case report and literature review about a rare entity. Cureus. 2021; 13(5):e14963.

https://doi.org/10.7759/cureus.14963

22. Sokoya M, Thomas CM, Eustaquio M. A rare collision tumor: tracheal sarcomatoid carcinoma invading a papillary thyroid carcinoma. Open Access Libr. J 2017; 4(12):16. https://doi.org/10.4236/oalib.1104012

23. Booya F, Sebo TJ, Kasperbauer JL, Fatourechi V. Primary squamous cell carcinoma of the thyroid: report of ten cases. Thyroid. 2006; 16(1):89-93.

https://doi.org/10.1089/thy.2006.16.89

24. Gul K, Ozdemir D, Ugras S, Inancli SS, Ersoy $\mathrm{R}$, Cakir B. Coexistent familial nonmultiple endocrine neoplasia medullary thyroid carcinoma and papillary thyroid carcinoma associated with RET polymorphism. Am J Med Sci. 2010; 340(1):60-63. https://doi.org/10.1097/MAJ.0b013e3181dfb24 5

25. Kleer CG, Giordano TJ, Merino MJ. Squamous cell carcinoma of the thyroid: an aggressive tumor associated with tall cell variant of papillary thyroid carcinoma. Mod Pathol. 2000; 13(7):742-746. https://doi.org/10.1038/modpathol.3880129

26. Darwish A, Satir AA, Hameed T, Malik S, Aqel N. Simultaneous medullary carcinoma, occult papillary carcinoma and lymphocytic thyroiditis. Malays J Pathol. 1995; 17(2):103-107.

27. Ljungberg $O$, Ericsson UB, Bondeson L, Thorell J. A compound follicular-parafollicular cell carcinoma of the thyroid: a new tumor entity? Cancer. 1983; 52(6):1053-1061. https://doi.org/10.1002/1097- 0142(19830915)52:6<1053::aid-

cncr2820520621>3.0.co;2-q

28. Gurkan E, Gurbuz Y, Tarkun I, Canturk Z, Cetinarslan B. Mixed medullary-papillary carcinoma of the thyroid: report of two cases and review of the literature. Indian $J$ Pathol Microbiol. 2014; 57(4):598-602. https://doi.org/10.4103/0377-4929.142684

29. Dikbas O, Duman AA, Guvendi GF. Medullary thyroid carcinoma and papillary thyroid carcinoma in the same patient as a collision tumour. Case Rep Endocrinol. 2019; 2019:4038628. https://doi.org/10.1155/2019/4038628

30. Brauckhoff M, Gimm O, Hinze R, Ukkat J, Brauckhoff K, Dralle H. Papillary thyroid carcinoma in patients with ret proto-oncogene germline mutation. Thyroid. 2002; 12(7):557561.

https://doi.org/10.1089/105072502320288393

31. Bounacer A, Wicker R, Caillou B, et al. High prevalence of activating ret proto-oncogene rearrangements, in thyroid tumors from patients who had received external radiation. Oncogene. 1997; 15(11):1263-1273. https://doi.org/10.1038/sj.onc.1200206

32. Ball DW. Medullary thyroid cancer: therapeutic targets and molecular markers. Curr Opin Oncol. 2007; 19(1):18-23. https://doi.org/10.1097/CCO.0b013e32801173e a

33. Vantyghem M-C, Pigny P, Leteurtre E, et al. Thyroid carcinomas involving follicular and parafollicular C cells: seventeen cases with characterization of RET oncogenic activation. Thyroid Off J Am Thyroid Asso.c 2004; 14(10):842-847. https://doi.org/10.1089/thy.2004.14.842

34. Weinhaeusel A, Scheuba C, Lauss M, et al. The influence of gender, age, and RET polymorphisms on C-cell hyperplasia and medullary thyroid carcinoma. Thyroid Off $J$ Am Thyroid Assoc. 2008; 18(12):1269-1276. https://doi.org/10.1089/thy.2008.0139. 